\title{
Fabrication of 3D complex micro-features used in bio-medical applications
}

\author{
Sandeep Kumar* and Akshay Dvivedi \\ Indian Institute of Technology Roorkee, Roorkee, Uttarakhand, 247667, India \\ *Email: sandeepkumar71@gmail.com
}

Miniaturization is the need of hour as it saves the space, time, material, and energy as well. Therefore the demand of miniaturized features, components and products is rapidly increasing in industries. These micro-products includes the features such as microholes, and microchannels which are extensively used in microfluidics [1], Bio-MEMS, microelectronic chip (heat transfer) applications [2] and Lab-on-a-chip. These microfeatures are fabricated by conventional as well as non-conventional methods. Conventional methods include micro drilling whereas nonconventional techniques are Lithography, Chemical etching, Embossing, LIGA and Laser fabrication etc. Glass is commonly used material in these kind of application because it is chemically inert to most of the materials, have good optical properties, hydrophilic, non-porous suitable for prototyping and inexpensive as well . However being a fragile material, it is very difficult to machine by conventional method. Whereas LIGA, lithography and chemical etching are unable to generate high aspect ratio microfeatures with good dimensional accuracy. Moreover these methods are time consuming and costly as well. Laser fabrication have the limitation to produce heat affected zone due to which workpiece properties change which further affect the useful life of product. Therefore there is a need to develop such process which can develop $3 \mathrm{D}$ complex micro-features at faster rate without altering the properties of workpiece.

Ultrasonic machining (USM) is one of the cost effective machining process used to machine all types of hard and brittle materials such as glass, quartz, ceramics, titanium etc. USM has wide applications in automobiles, electronics, MEMS, and optics etc. This process is best suited for those materials which have low ductility and hardness greater than 60 HRC. USM comprises of power supply, transducer, horn, tool and abrasive slurry. In micro-USM, material is removed from the workpiece surface by the hammering and impact action of abrasives followed by micro-chipping, cavitation erosion and chemical reaction. This process also has limitations such as low aspect ratio and high tool wear due to inefficient circulation of abrasive slurry in between microtool and workpiece which restrict its use in micromachining of deep cavities. This limitation can be eliminated by providing rotation to the tool. In this article, preliminary studies were conducted on borosilicate glass material by using rotary tool ultrasonic micromachining. Microholes and microchannels were fabricated. Further, the results were compared with stationary tool micro-USM. The results revealed that high aspect ratio micro-holes and microchannels with good dimensional accuracy can be developed by using rotary tool ultrasonic micro machining process. The schematic representation of rotary tool micro-USM setup is shown in Figure 1.

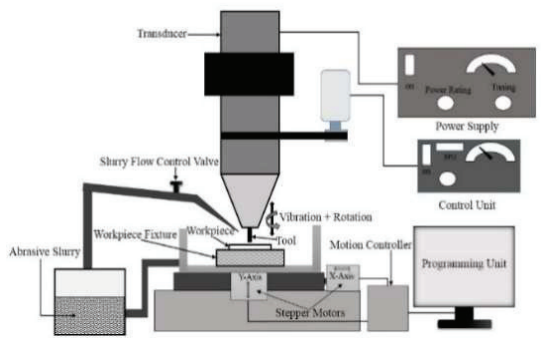

Figure 1: Schematic of rotary tool micro-USM

In rotary tool micro-USM, due to the rotation of the micro-tool, the abrasive particles did not stuck in between the tool and workpiece. As a result of that deep micro-features with good surface finish were developed. Moreover tool wear was also reduced by providing rotation to the tool which resulted in good dimensional accuracy of the generated micro-features. Micro-holes of diameter $650 \mu \mathrm{m}$ and depth upto $2000 \mu \mathrm{m}$ and different types of 3D complex shape microchannels such as Serpentine, Zig-Zag, Double Y etc. fabricated successfully in the proposed study.

\section{References}

1. P. Yao, J. S. Garrett, D.W. Prather, Journal of Microelectromechanical Systems 14 (2005) 799-805. 2. H.Y. Wu, P. Cheng, International Journal of Heat and Mass Transfer 46 (2003) 2547-2556. 